\title{
Novel rodent model for simulation of sylvian fissure dissection and cerebrovascular bypass under subarachnoid hemorrhage conditions: technical note and timing study
}

\author{
Avital Perry, MD, ${ }^{1}$ Christopher S. Graffeo, MD, ${ }^{1}$ Lucas P. Carlstrom, MD, ${ }^{1}$ \\ William J. Anding, ${ }^{2}$ Michael J. Link, MD, ${ }^{1,3}$ and Leonardo Rangel-Castilla, MD ${ }^{1,4}$ \\ Departments of ${ }^{1}$ Neurologic Surgery, ${ }^{2}$ Education, ${ }^{3}$ Otolaryngology-Head and Neck Surgery, and ${ }^{4}$ Radiology, Mayo Clinic, \\ Rochester, Minnesota
}

\begin{abstract}
OBJECTIVE Sylvian fissure dissection following subarachnoid hemorrhage (SAH) is a challenging but fundamental skill in microneurosurgery, and one that has become increasingly difficult to develop during residency, given the overarching management trends. The authors describe a novel rodent model for simulation of sylvian fissure dissection and cerebrovascular bypass under SAH conditions.

METHODS A standardized microvascular anastomosis model comprising rat femoral arteries and veins was used for the experimental framework. In the experimental protocol, following exposure and skeletonization of the vessels, extensive, superficial (1- to 2-mm) soft-tissue debridement was conducted and followed by wound closure and delayed reexploration at intervals of 7,14 , and 28 days. Two residents dissected 1 rat each per time point $(n=6$ rats), completing vessel skeletonization followed by end-to-end artery/vein anastomoses. Videos were reviewed postprocedure to assess scar score and relative difficulty of dissection by blinded raters using 4-point Likert scales.
\end{abstract}

RESULTS At all time points, vessels were markedly invested in friable scar, and exposure was subjectively assessed as a reasonable surrogate for sylvian fissure dissection under SAH conditions. Scar score and relative difficulty of dissection both indicated 14 days as the most challenging time point.

CONCLUSIONS The authors' experimental model of femoral vessel skeletonization, circumferential superficial softtissue injury, and delayed reexploration provides a novel approximation of sylvian fissure dissection and cerebrovascular bypass under SAH conditions. The optimal reexploration interval appears to be 7-14 days. To the authors' knowledge, this is the first model of SAH simulation for microsurgical training, particularly in a live animal system.

https://thejns.org/doi/abs/10.3171/2018.11.FOCUS18533

KEYWORDS simulation; education; cerebrovascular; bypass; subarachnoid hemorrhage; aneurysm; rat

$\mathrm{T}$ HE landscape of neurosurgical cerebrovascular training is shifting dynamically, as a number of forces have collectively resulted in decreased microsurgical operative experience, including duty-hour restrictions, improvements in endovascular and radiosurgical technology and experience, and trends in litigation. ${ }^{5,10,11,17,20,23}$ In order to increase training efficiency and decrease the risk of compromising patient safety, numerous avenues have been explored for the development of neurosurgical simulators, including models based on large and small animals, perfused human or animal cadavers, placenta, virtual reality, augmented reality, haptic controllers, molded or cast synthetics, 3D printing, and many others. $13,4,6-9,14-16,18,21,22,24-27,29$

The development of a sophisticated microsurgical skill set-the pillar of many neurosurgical niches-is simultaneously more susceptible to erosion in the current clinical context and also more challenging to meaningfully replicate in a simulation environment, given that few highfidelity models have been developed or validated. Open cerebrovascular neurosurgery epitomizes this conflict, given the continuing decline in case volumes, coupled with

ABBREVIATIONS SAH = subarachnoid hemorrhage 
high case complexity, significant potential for morbidity, and increased technical difficulty, particularly following subarachnoid hemorrhage (SAH). Of particular interest, cerebral revascularization-more commonly known as bypass - remains a critical tool in the cerebrovascular armamentarium, but it is one that potentially compromises a healthy arterial distribution, and therefore presents a major hurdle in ensuring technical proficiency among graduating residents and fellows without subjecting patients to undue risk..$^{2,12,28}$ In contrast to most neurosurgical simulators, a number of successful models have been developed for anastomosis training using rodent artery and vein reconstructions, largely in coordination with plastic, otolaryngology, urology, and hand surgery training programs, given the relative prominence of microanastomosis procedures in their routine practice..$^{19}$

Sylvian fissure dissection remains a fundamental microneurosurgical skill, yet no model has been developed to simulate SAH conditions. Correspondingly, our goal in the present pilot study was to leverage the high-fidelity microsurgical simulation provided by rodent model anastomosis training in combination with a novel methodology for inducing SAH-like tissue, in order to develop and test a new and potentially high-yield approach to teaching both sylvian fissure dissection and cerebral revascularization techniques.

\section{Methods}

The study setting was our microvascular education center, and we used a standardized rat femoral artery and vein anastomosis model as the control (Fig. 1A). Standard microsurgical dissection technique was employed to expose the femoral vessels, including sharp skin incision, inferolateral mobilization and fishhooking of the epigastric fat pad and vessels, suture retraction of the inguinal ligament, and isolation and skeletonization of the femoral artery and vein from the profunda femoris to their distal bifurcations (Fig. 1B).

In the control model, the vascular clamps were then positioned; the vessel was opened, dilated, and injected with heparinized saline; and basic end-to-end artery and vein microvascular anastomoses were completed. Following wound closure, the rat recovered from anesthesia, and the wound was explored $>24$ hours later by an independent study staff (W.J.A.) to determine bypass patency.

In the experimental model, initial exposure of the femoral artery and vein was carried out by an independent, nonsubject study staff member (W.J.A.), who then additionally dissected and extensively debrided, to a depth of 1-2 mm, the surrounding soft tissues. The wound was closed, and the rat was returned to routine care for a predetermined time interval ( 7,14 , or 28 days). Two rats were included at each time point, one each being dissected by each of the two resident subjects (A.P. and C.S.G.), resulting in a total of 6 experimental animals in this pilot study, 3 per resident. At the time of this study, each resident had independently completed $>100$ anastomoses using our facility and under conditions akin to the control model, with patency rates $>95 \%$. At the specified time points, the wound was reexplored and the resident was instructed to fully isolate

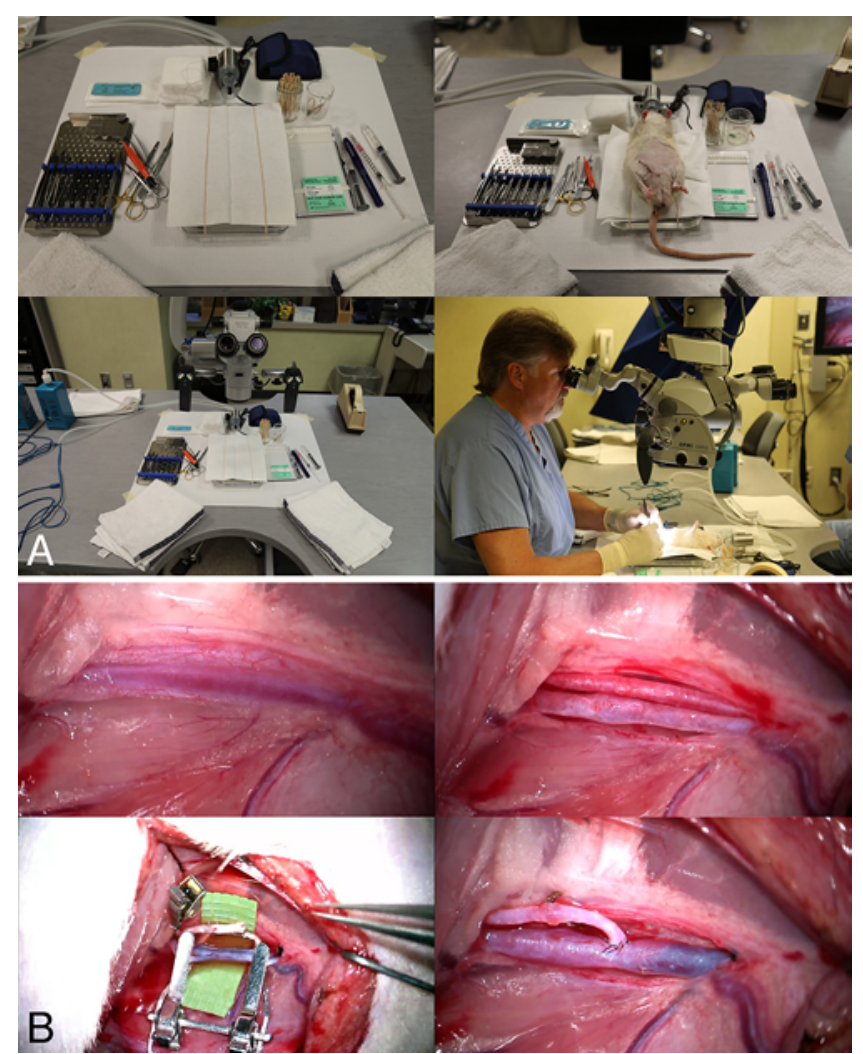

FIG. 1. A: Control and experimental models were explored in our institutional microvascular skills laboratory using as a framework routine rat femoral artery and vein anastomoses. Facilities are equipped with inhalational anesthesia, operating room-equivalent microsurgery instruments, and Carl Zeiss OPMI VARIO microscopes. B: In the control model, femoral vessels were exposed, skeletonized, clamped, ligated, and anastomosed. The example end-to-side artery-to-vein anastomosis is shown for demonstrative purposes only; control animals in the study protocol underwent end-to-end artery and vein anastomoses, as described.

and skeletonize the femoral vessels and subsequently complete arterial and venous end-to-end anastomoses. Reexploration and bypass procedures were recorded on video for subsequent review. Postprocedure, 3 blinded reviewers ( 2 residents [A.P. and C.S.G.] and 1 independent, non-subject study staff [L.P.C.]) subjectively graded each of 6 videos with a scar score and relative difficulty of dissection, using 4-point Likert scales for degree of change attributable to altered environmental factors (none, mild, moderate, or severe). As in the control model, graft patency was independently assessed at $>24$ hours postbypass.

All pertinent components of this study were performed with Institutional Animal Care and Use Committee approval and in accordance with institutional protocols and regulations regarding educational and research activities involving animals. General provisions applicable to our experimental model included those ensuring good animal welfare, appropriate pain management, daily wound care, and humane sacrifice, as well as a host of specific institutional protocols for managing the array of potential issues that might arise during the postprocedure presacrifice period. 

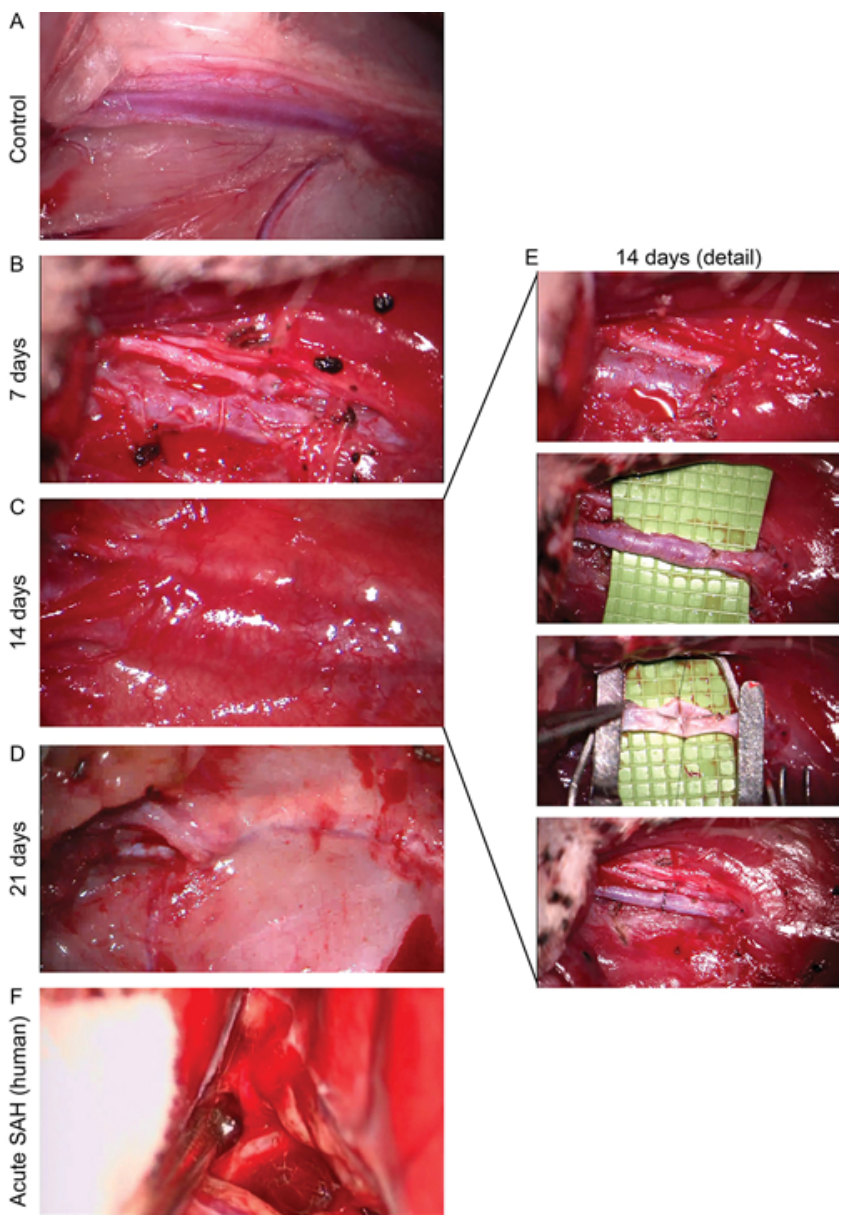

FIG. 2. Periprocedural images acquired as video stills from control and experimental trials are shown at time of wound reopening but prior to vessel dissection/skeletonization in controls $(\mathbf{A})$ and at 7-day (B), 14day (C), and 21-day (D) time points. E: Several stepwise details from the 14-day time point are included to highlight the contrast between experimental and control conditions, with particular attention to the scar density and tissue friability. F: A sample frame taken from an intraoperative microscope video of sylvian fissure dissection following aneurysmal $\mathrm{SAH}$ is shown for comparison.

\section{Results}

On inspection following execution of the study protocol, experimental animals at all time points demonstrated femoral vessels that were markedly invested in heterogeneous fibrosis, adhesions, and layers of both superficial and circumferential scar tissue, as compared to control rats (Fig. 2A-E). Of particular note, the scar was observed to be highly friable throughout the dissection. Subjective assessment of perivascular scar formation indicated reasonable similarity to sylvian fissure dissection following SAH (Fig. 2F).

Resident dissections of experimental model rats at all time points demonstrated a dramatic increase in fibrosis, adhesions, and investment of vessels within scar, resulting in a significantly more challenging dissection at all time points relative to control animals; blinded review concluded that the largest increase in relative difficulty of dissection occurred at 14 days (Fig. 3A). Similarly, mild to
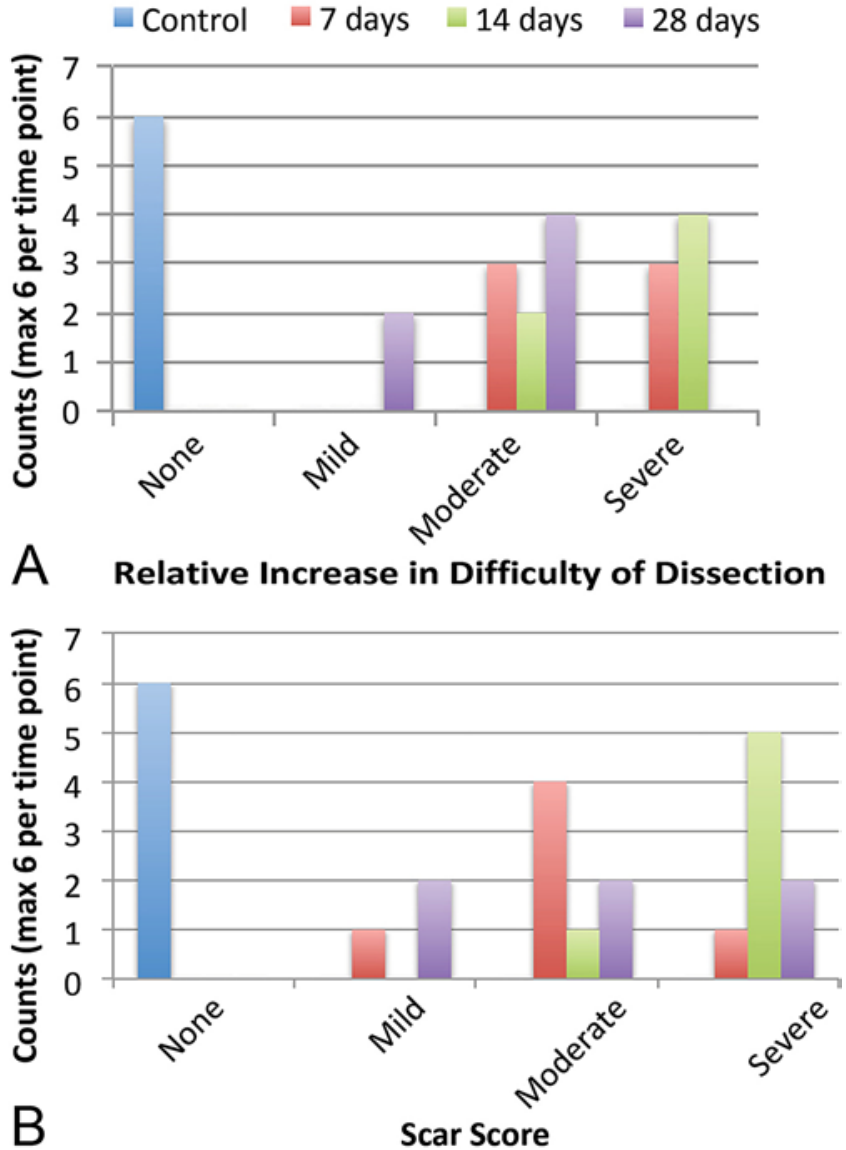

FIG. 3. Bar graphs highlight the relative increase in difficulty of dissection $(\mathbf{A})$ and scar score $(\mathbf{B})$ counts assigned by blinded video reviewers using a 4-point Likert scale (none, mild, moderate, and severe). Ideal model timing for a maximally challenging and high-fidelity simulation most likely falls within the 7- to 14-day range.

severe scar scores were recorded for all time points, with severe scores assigned in 5 of 6 assessments at 14 days and 4 of 6 assessments at 7 days (Fig. 3B).

Bypass completion times (e.g., clamp-on times) during experimental conditions were 1.5 - to 2 -fold longer than the approximate 20-minute mean time established for each resident during cumulative preceding training exercises. On postbypass assessment of 12 experimental anastomoses in 6 animals, 11 remained patent (92\%); 1 venous anastomosis thrombosed that had been performed at the 7-day time point, which on external and internal inspection revealed no intimal flap, vessel wall injury, intraluminal adventitia, diameter mismatch, bite size irregularity, excessive suture placement, asymmetrical suture spacing, or other obvious underlying technical error. There were no animal deaths, site hemorrhages, or other unanticipated complication or protocol deviation.

\section{Discussion}

We have described a novel, effective, reproducible, and easy-to-execute rodent model for simulation of sylvian fissure dissection and cerebrovascular bypass un- 
der SAH conditions. This is a key area for expansion of high-fidelity simulators and related training resources, particularly considering the dramatic increase in the difficulty of microsurgical dissection that results from SAH and the relative paucity of opportunities for direct operative experience with SAH during residency. This problem is anticipated to become more severe in the future given the shifting landscape of open versus endovascular management of ruptured aneurysms, further underscoring the need for aggressive development of supplemental educational tools. ${ }^{1,13}$

Based on this index experience, we preliminarily recommend a 2-week time point as the best opportunity to simulate SAH-like operative conditions; however, given our observations of marked increases in both relative difficulty and scar score at 7 and 14 days, it may ultimately prove equally efficacious and significantly more efficient to use 7-day protocols for future simulation exercises. By contrast, at 28 days the scar formation has evolved and organized to such an extent that the conditions, while still more challenging than the control model, are regressing toward baseline, with decreased tissue friability, thinning of the superficial scar tissue, and decreased adhesiveness between the vessels and the scar. Correspondingly, time points beyond 14 days are not recommended for future simulations, as they are anticipated to be both lower yield and higher cost. Further testing is required to validate this conclusion and clarify our findings in a larger cohort.

Excellent foundational studies have been carried out on the role of rodent-model simulation of microvascular anastomosis, particularly by subspecialties with a significant investment in ensuring that high-quality vascularized free-flap operations are integrated into their routine practices and basic resident curricula. ${ }^{19}$ Our work adapts these pedagogical frameworks to the needs of neurosurgical training, and it incorporates a novel methodology for generating operative conditions akin to $\mathrm{SAH}-\mathrm{a}$ highcomplexity, high-stakes clinical context that at present has no other reliable or robust simulation model.

The specific goals of incorporating a microvascular anastomosis into the proposed training model warrant detailed consideration, particularly given that the experimental conditions would not be expected to have a significant impact on the difficulty of the bypass itself, as compared to the standard rat femoral vessels model. The most important role of the bypass is to set a clear endpoint for the dissection, such that all participants will be required to fully expose and skeletonize the femoral vessels in order to prepare for and complete the bypass. In parallel, the experimental dissection is intended to be challenging and fatiguing, and so placing a high-stakes exercise (e.g., bypass) at the conclusion of that phase of the model valuably simulates the more common clinical scenario, in which the potentially stressful and taxing exposure of the ruptured aneurysm dome is followed by the high-stakes but arguably less challenging clip application.

Furthermore, in addition to added value in the simulation itself, the inclusion of a bypass following the experimental model dissection has value as an objective metric-both for the individual trainee and for future research purposes. In an adequate sample of trainees demonstrating diverse levels of skill and experience, we anticipate that a lower patency rate will be observed as compared to the trainee's baseline and in the overall experimental sample compared with matched controls - a series of critical research questions we intend to assess in a future randomized follow-up study. Finally, given the overall cost of the model, the inclusion of the bypass ensures that maximum value is extracted from the exercise by each trainee, which we anticipate will prove useful in a range of other microsurgical procedures.

Our study is limited in several key ways, most significantly the small cohort of residents $(\mathrm{n}=2)$ and the relative inexperience of any residents with acute $\mathrm{SAH}$, which correspondingly limits their ability to accurately benchmark the clinical fidelity of the experimental model. Further limitations can be conceptually grouped as pertaining to the model and its implementation or to the study design itself.

With respect to the model, although our preliminary experience suggests that the experimental conditions provide a reasonable analog for the superficial dissection of sylvian vessels under SAH conditions, this is but one aspect of the inherent challenges of these operations. More specifically, while a longitudinal exposure of the vessels, including their isolation from the surrounding acutely inflamed, swollen, and scarred soft tissue is provided, the anatomical constraints of the rat profunda femoris are such that there is little to no simulation of dissecting at a depth more deeply in the fissure. In future iterations of the model, we plan to incorporate permutations in which the distance between the operator and the vessels will be artificially augmented (e.g., forcing trainees to operate through a ring that is several inches deep); however, even with such modifications, the simulation will never truly replicate all the challenges of sylvian dissection. Another important limitation of the model is financial, as the implementation of such a training experience mandates the existence or creation of a microvascular training facility. Costs associated with implementation of a model such as ours would include both general laboratory overhead and additional expenses pertinent to the experimental conditions. Based on institutional expense reports, we estimate that maintaining a fully functioning microvascular anastomosis laboratory with baseline utilization of 1 trainee per day over a 50 -week period to be approximately $\$ 80,000$, which includes facilities, personnel, suture and disposable supplies, and animal purchase, shipping, housing, and care. Further routine expenses (e.g., animal and surgical supply costs for additional trainees) are approximated at $\$ 65$ per day per trainee. Following acquisition, all routine animal care expenses are estimated at $\$ 1$ per day per rat; correspondingly, although each individual completing the experimental model would incur a total cost of approximately $\$ 80$, the differential cost versus standard protocol would be no more than $\$ 5$ to $\$ 15$ per trainee, depending on the time interval elected between initial exposure and dissection.

Regarding the limitations of our assessment strategy, the scales designed for this experimental model were subjective and were applied retrospectively, albeit in a blinded 
fashion. Furthermore, in this pilot iteration of the study, the small sample limits our ability to objectively study the assessments via implementation of an interrater reliability measurement. Addressing this question will be a key focus of the phase 2 experiments, in which we will randomize a larger number of variably skilled trainees to experimental versus control conditions. Although the gestalt parameters regarding relative difficulty, including operator assessment, observer assessment, and operative time, were all reasonable, and their agreement with one another gives us confidence with respect to their appropriateness as surrogate metrics, it would be more favorable to objectively assess the impact of the experimental conditions on operative performance-for example, using ergonomic monitors to assess efficiency of movement, tremor, and so on. Notwithstanding, and in spite of the obvious limitations inherent in pilot projects such as the described study, we have outlined and successfully executed a new protocol that our preliminary data suggest will provide a very helpful adjunct to resident- and fellow-level microsurgical training for $\mathrm{SAH}$.

\section{Conclusions}

In this proof-of-concept trial for a novel simulation procedure, our model subjectively demonstrated the efficacy of a new experimental protocol for generating SAH-like microsurgical conditions in a rodent model. Preliminary subjective data indicate that our model may provide a robust and easily incorporated modality for simulation of this uncommon but critically important and technically challenging clinical condition. Based on our initial timing study, we recommend focused assessment in a larger cohort of the 14-day protocol, with consideration given for a 7-day alternative procedure that may improve overall efficiency of the model without a sacrifice in educational value. Simulation will likely constitute a cornerstone of microneurosurgery education in the future, and it is critical that our community develop, assess, and integrate models such as this into formal resident and fellow didactic curricula to ensure that the core technical skill set of our subspecialties continues to be well developed in the next generation of neurosurgeons.

\section{References}

1. Abla AA, Lawton MT: Three-dimensional hollow intracranial aneurysm models and their potential role for teaching, simulation, and training. World Neurosurg 83:35-36, 2015

2. Abla AA, Lawton MT, Spetzler RF: The art of basilar apex aneurysm surgery: is it sustainable in the future? World Neurosurg 82:e51-e53, 2014

3. Alaraj A, Luciano CJ, Bailey DP, Elsenousi A, Roitberg BZ, Bernardo A, et al: Virtual reality cerebral aneurysm clipping simulation with real-time haptic feedback. Neurosurgery 11 (Suppl 2):52-58, 2015

4. Baskaran V, Štrkalj G, Štrkalj M, Di Ieva A: Current applications and future perspectives of the use of 3D printing in anatomical training and neurosurgery. Front Neuroanat 10:69, 2016

5. Bohm PE, Arnold PM: Simulation and resident education in spinal neurosurgery. Surg Neurol Int 6:33, 2015

6. Breimer GE, Haji FA, Bodani V, Cunningham MS, LopezRios A-L, Okrainec A, et al: Simulation-based education for endoscopic third ventriculostomy: a comparison between virtual and physical training models. Oper Neurosurg (Hagerstown) 13:89-95, 2017

7. Colpan ME, Slavin KV, Amin-Hanjani S, Calderon-Arnuphi M, Charbel FT: Microvascular anastomosis training model based on a Turkey neck with perfused arteries. Neurosurgery 62 (5 Suppl 2):ONS407-ONS411, 2008

8. John NW, Phillips NI, Cenydd LA, Coope D, Carleton-Bland N, Kamaly-Asl I, et al: A tablet-based virtual environment for neurosurgery training. Presence Teleoperators Virtual Environ 24:155-162, 2015

9. John NW, Phillips NI, Cenydd LA, Pop SR, Coope D, Kamaly-Asl I, et al: The use of stereoscopy in a neurosurgery training virtual environment. Presence Teleoperators Virtual Environ 24:289-298, 2016

10. Kirkman MA, Ahmed M, Albert AF, Wilson MH, Nandi D, Sevdalis N: The use of simulation in neurosurgical education and training. A systematic review. J Neurosurg 121:228246, 2014

11. Konakondla S, Fong R, Schirmer CM: Simulation training in neurosurgery: advances in education and practice. Adv Med Educ Pract 8:465-473, 2017

12. Lawton MT: Basilar apex aneurysms: surgical results and perspectives from an initial experience. Neurosurgery 50:110,2002

13. Lindgren A, Vergouwen MD, van der Schaaf I, Algra A, Wermer M, Clarke MJ, et al: Endovascular coiling versus neurosurgical clipping for people with aneurysmal subarachnoid haemorrhage. Cochrane Database Syst Rev 8:CD003085, 2018

14. McGuire LS, Alaraj A: Competency assessment in virtual reality-based simulation in neurosurgical training, in Alaraj A (ed): Comprehensive Healthcare Simulation: Neurosurgery. Cham, Switzerland: Springer, 2018, pp 153-157

15. Oliveira Magaldi M, Nicolato A, Godinho JV, Santos M, Prosdocimi A, Malheiros JA, et al: Human placenta aneurysm model for training neurosurgeons in vascular microsurgery. Neurosurgery 10 (Suppl 4):592-601, 2014

16. Rangwala S, Arnone G, Charbel FT, Alaraj A: Ventriculostomy simulation in neurosurgery, in Alaraj A (ed): Comprehensive Healthcare Simulation: Neurosurgery. Cham, Switzerland: Springer, 2018, pp 17-28

17. Rehder R, Abd-El-Barr M, Hooten K, Weinstock P, Madsen JR, Cohen AR: The role of simulation in neurosurgery. Childs Nerv Syst 32:43-54, 2016

18. Sadasivan C, Lieber BB, Woo HH: Physical simulators and replicators in endovascular neurosurgery training, in Alaraj A (ed): Comprehensive Healthcare Simulation: Neurosurgery. Cham, Switzerland: Springer, 2018, pp 29-45

19. Shurey S, Akelina Y, Legagneux J, Malzone G, Jiga L, Ghanem AM: The rat model in microsurgery education: classical exercises and new horizons. Arch Plast Surg 41:201-208, 2014

20. Suri A, Tripathi M, Bettag M, Roy TS, Lalwani S: Simulation based skills training in neurosurgery and contemporary surgical practices. Ann Natl Acad Med Sci (India) 52:5675,2016

21. Tham TM, Costantino PD, Rahman L, Lotzky G: 3D skull reconstruction with selective laser sintering for endoscopic cranial base surgical simulation and training. J Neurol Surg B Skull Base 77 Suppl:133, 2016 (Abstract)

22. Thawani JP, Ramayya AG, Abdullah KG, Hudgins E, Vaughan K, Piazza M, et al: Resident simulation training in endoscopic endonasal surgery utilizing haptic feedback technology. J Clin Neurosci 34:112-116, 2016

23. Tripathi M, Yagnick NS, Mohindra S, Batish A, Gupta SK: Sully, simulation, and neurosurgery. World Neurosurg 118:400-401, 2018

24. Vakharia VN, Vakharia NN, Hill CS: Review of 3-dimen- 
sional printing on cranial neurosurgery simulation training. World Neurosurg 88:188-198, 2016

25. Vite ST, Velasco CD, Valencia AFH, Lomelí JSP, Castañeda MÁP: Virtual simulation of brain Sylvian fissure exploration and aneurysm clipping with haptic feedback for neurosurgical training, in De Paolis LT, Bourdot P (eds): International Conference on Augmented Reality, Virtual Reality and Computer Graphics. Cham: Switzerland: Springer, 2018, pp 230-238

26. Waran V, Narayanan V, Karuppiah R, Pancharatnam D, Chandran H, Raman R, et al: Injecting realism in surgical training-initial simulation experience with custom 3D models. J Surg Educ 71:193-197, 2014

27. Waran V, Narayanan V, Karuppiah R, Thambynayagam HC, Muthusamy KA, Rahman ZAA, et al: Neurosurgical endoscopic training via a realistic 3-dimensional model with pathology. Simul Healthc 10:43-48, 2015

28. Yoon S, Burkhardt JK, Lawton MT: Long-term patency in cerebral revascularization surgery: an analysis of a consecutive series of 430 bypasses. J Neurosurg [epub ahead of print August 24, 2018. DOI: 10.3171/2018.3.JNS172158]

29. Zada G, Bakhsheshian J, Pham M, Minneti M, Christian E, Winer J, et al: Development of a perfusion-based cadaveric simulation model integrated into neurosurgical training: feasibility based on reconstitution of vascular and cerebrospinal fluid systems. Oper Neurosurg (Hagerstown) 14:72-80, 2018

\section{Disclosures}

The authors report no conflict of interest concerning the materials or methods used in this study or the findings specified in this paper.

\section{Author Contributions}

Conception and design: all authors. Acquisition of data: RangelCastilla, Perry, Graffeo, Anding. Analysis and interpretation of data: Perry, Graffeo, Carlstrom. Drafting the article: Perry, Graffeo. Critically revising the article: Rangel-Castilla, Perry, Graffeo, Carlstrom, Link. Reviewed submitted version of manuscript: Perry, Graffeo. Administrative/technical/material support: Rangel-Castilla, Link. Study supervision: Rangel-Castilla, Link.

\section{Supplemental Information \\ Videos \\ Video Abstract. https://vimeo.com/310207859.}

\section{Correspondence}

Leonardo Rangel-Castilla: Mayo Clinic, Rochester, MN. rangelcastilla.leonardo@mayo.edu. 